\title{
2D kinematics of the edge-on spiral galaxy ESO 379-006
}

\author{
M. Rosado $^{1}$, R. F. Gabbasov ${ }^{1}$, P. Repetto ${ }^{1}$, I. Fuentes-Carrera ${ }^{2}$, \\ P. Amram ${ }^{3}$, M. Martos ${ }^{1}$ and O. Hernandez ${ }^{3,4}$ \\ ${ }^{1}$ Instituto de Astronomía, Universidad Nacional Autónoma de México, México \\ Apartado Postal 70-264, CP04510, México, D. F. \\ email: margarit@astro.unam.mx \\ ${ }^{2}$ Escuela Superior de Física y Matemáticas, IPN, México \\ Box 515, SE-75120 Uppsala, Sweden \\ email: hoefner@astro.uu.se \\ ${ }^{3}$ Laboratoire d'Astrophysique de Marseille, France \\ ${ }^{4}$ Université de Montréal, Canada
}

\begin{abstract}
We present a kinematical study of the marginally edge-on galaxy ESO 379-006 . With Fabry-Perot spectroscopy at $\mathrm{H} \alpha$ we obtain velocity maps, the radial velocity field, and position-velocity diagrams parallel to the major and to the minor axis of the galaxy. We build the rotation curve of the galaxy and discuss the role of projection effects. The twisting of isovelocities in the radial velocity field of the disk of ESO 379-006 as well as a kinematical asymmetry found in the position-velocity diagrams parallel to the minor axis suggest the existence of noncircular motions that can be modeled by including a radial inflow besides the rotation motion. Extraplanar Diffuse Ionized gas was detected in this galaxy both from the images and from its kinematics. It is possible that the diffuse gas is lagging in rotation.
\end{abstract}

\section{Results}

The Fabry-Perot data were obtained at the ESO $3.6 \mathrm{~m}$ telescope with the CIGALE instrument. These data allowed us to construct velocity maps, the radial velocity field and position-velocity diagrams (PVDs) parallel to the major and minor axis of the galaxy ESO 379-006. Rotation Curves (RCs) were obtained from the intensity peaks of the velocity profiles (barycentric) and by the Envelope-Tracing Method. A comparison between them shows that projection effects are important in the inner regions of the galaxy. We have modeled this galaxy using the GALMOD task of GYPSY. We explored almost all the galactic parameters such as inclination, vertical scale height, velocity dispersion, systemic velocity, center, etc. and the model that fits the best the important features derived from the observations has an inclination of 82.5 degrees and a vertical scale height of 2 arcsec among the main parameters, together with the inclusion of an additional velocity, in the radial direction, corresponding to an inflow (Fig. 1). In addition, the PVD along the major axis shows some low velocity extensions ("the beard") that can be interpreted either as extraplanar Ionized Diffuse Gas (from which we know about its existence and detection in this particular galaxy) or as projection effects due to the high inclination of the galaxy. 

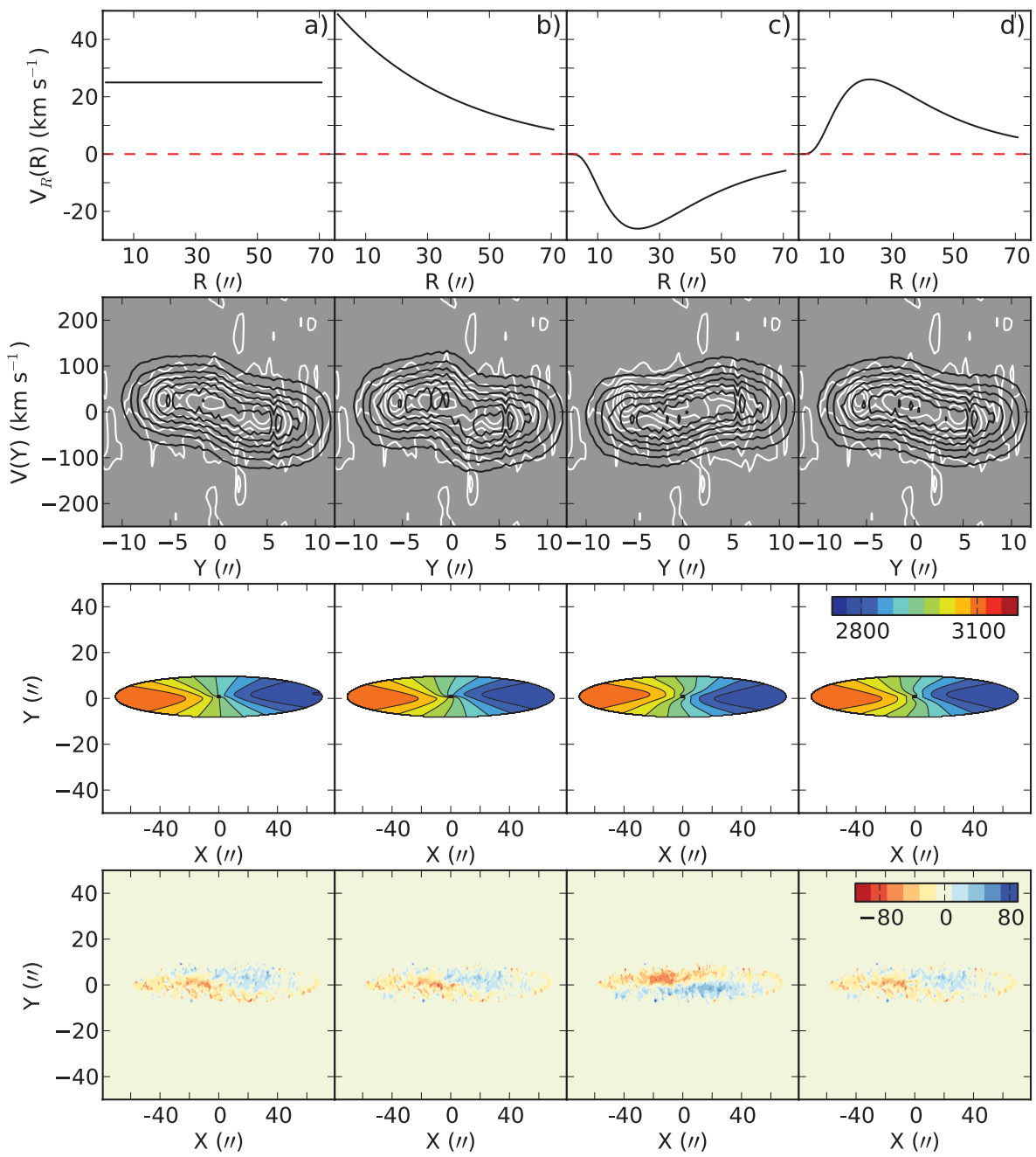

Figure 1. The radial motion distribution (top row), a comparison of modeled (black contours) and observed (white contours) minor axis PVD (second row), synthetic velocity fields (third row) and the residual velocity fields (bottom row) for models with a constant inflow (a), exponential decaying inflow (b), lognormal outflow (c) and the lognormal inflow (d).

\section{Acknowledgements}

This paper was done with financial support from grants P-82389 from CONACYT. 